\title{
Diagnosis and Treatment of Multidrug-Resistant Tuberculosis in Developed and Developing Countries: Finally Towards Equality?
}

\author{
Rafael Laniado-Laborín ${ }^{*}, 1$, Domingo J. Palmero ${ }^{2}$ and José A. Caminero-Luna ${ }^{3}$ \\ ${ }^{1}$ Clinica y Laboratorio de Tuberculosis, Hospital General de Tijuana, ISESALUD, Mexico \\ ${ }^{2}$ Hospital Dr. F.J. Muñiz, Buenos Aires, Argentina \\ ${ }^{3}$ Servicio de Neumología, Hospital de Gran Canaria Dr. Negrín, Las Palmas de Gran Canaria, España
}

\begin{abstract}
Tuberculosis TB is considered a sobering example of inequity. It is a disease predominantly of the socially and economically disadvantaged. This neglect is evidenced by lack of political support, scarce financial resources for TB programs, and little or no leadership.

The emergence and spread of multidrug-resistant tuberculosis (MDR-TB) is threatening global TB control and it represents a major challenge for clinical care and operational management. Worldwide, $3.7 \%$ of new cases and $20 \%$ of previously treated cases are estimated to have MDR-TB. Unfortunately, since only a quarter of patients with MDR-TB are treated according to established standards and the proportion of treatment success does not exceed $50 \%$, extensively drugresistant tuberculosis (XDR-TB) has already been reported in 84 countries and totally drug resistant cases have been recently described.

In most low- and middle-income countries drug sensitivity testing is not performed for new cases or for most patients requiring retreatment. Therefore, patients with underlying drug resistance will receive retreatment with first line drugs and can be predicted to have higher rates of failure and relapse.

The development of genotypic methods has generated a genuine revolution in the diagnosis of DR-TB. The polymerase chain reaction allows for the specific identification of $M$. tuberculosis and the detection of drug resistance in a matter of hours instead of weeks. Unfortunately these techniques are expensive and not available in most high burden countries.

Despite the enormous number of cases of TB worldwide, the therapeutic arsenal to treat this disease continues to be very limited, especially for cases with extensive drug resistance. However, for the first time in decades, the pipeline of new anti-TB agents is now growing again inasmuch as new drugs and combination of drugs with interesting potential efficacy to treat TB, MDR-TB and XDR-TB have appeared during the last few years.
\end{abstract}

Keywords: MDR-TB, social inequity, diagnosis, treatment.

\section{INTRODUCTION}

The emergence and spread of multidrug-resistant tuberculosis (MDR-TB, defined as the simultaneous resistance at least to isoniazid and rifampin), an emergent problem increasing worldwide year by year, is threatening global TB control and it represents a major challenge for clinical care and operational management $[1,2]$.

An estimated 5\% of all new cases of tuberculosis diagnosed in 2006 were multidrug resistant. This represents an increase of $12 \%$ since 2004 and 56\% since 2000 [3-5]. Globally in 2011, there were an estimated 630, 000 cases of MDR-TB (range, 460,000- 790,000) among the world's 12 million prevalent cases of TB [6]. While the number of new cases of MDR-TB notified in the 27 high MDR-TB burden countries is increasing and reached almost 60,000 worldwide in 2011 , this is only one in five $(19 \%)$ of the notified TB patients estimated to have MDR-TB. In the two countries with the largest number of cases, India and China, the figure

*Address correspondence to this author at the Emiliano Zapata 1423, Zona Centro. Tijuana, CP 22000, México; Tel/Fax: 011(52)686-5626;

E-mail: rlaniado@uabc.edu.mx is less than one in ten [6]. Worldwide, 3.7\% of new cases and $20 \%$ of previously treated cases were estimated to have MDR-TB. India, China, the Russian Federation and South Africa have almost $60 \%$ of the world's cases of MDR-TB. The highest proportions of TB patients with MDR-TB are in Eastern Europe and central Asia.

Unfortunately, since only a quarter of patients with MDR-TB are treated according to established standards and the proportion of treatment success does not exceed 50\% [6], extensively drug-resistant tuberculosis (XDR-TB), defined as MDR-TB plus resistance to any antituberculosis fluorquinolone and any second line injectable drug (amikacin, kanamycin or capreomycin) has already been reported in 84 countries; the average proportion of MDR-TB cases with XDR-TB is $9.0 \%$ [6]. Totally drug resistant cases (i.e. Mycobacterium tuberculosis strains with resistance to all the drugs tested) have been recently described in Italy, Iran, and India [7, 8].

Unfortunately, as it happens with drug susceptible TB, most of the MDR-TB cases occur in developing countries, with limited or no resources for diagnosis or access to second line drugs for treatment of resistant cases. As would 
be expected, countries with ample resources for diagnosis and treatment of MDR-TB cases are those with the lowest rates of drug resistant cases. A global effort towards equality is an urgent and essential need if we are going to attain control of this MDR-TB pandemic.

\section{HISTORY AND PATHOGENESIS OF MDR-TB}

Resistance to TB drugs emerged soon after their introduction in the 1940's, but the problem was considered as minor since it accounted for only a small proportion of treatment failures. Furthermore, clinical trials suggested that empirical standard treatment without routine baseline testing for drug susceptibility produced outcomes similar to those obtained where such testing was applied and individualized treatment was given [9]. The introduction of rifampicin in the early 1970's allowed for the first time the implementation of ambulatory short-course chemotherapy, a regimen that included three or four drugs given over six to nine months $[10,11]$. This generated the notion that finally TB could be, like smallpox, not only controlled but also eliminated worldwide, which led to budgetary reductions for TB control programs, including those from developed countries. As should have been expected, tuberculosis resurged during the 1990's even in industrialized countries [12]. As a consequence, the World Health Organization (WHO) declared TB as a global emergency in 1993, focusing especially on developing countries where $95 \%$ of cases occurred [13].

The primary cause of an uncontrolled and increasing TB epidemic worldwide has been the neglect of TB control programs. This neglect is evidenced by lack of political support, scarce financial resources, and little or no leadership [11]. As mentioned, by the early 1990's the incidence of TB had increased, following not only budgetary cuts, but also the growing HIV epidemic and prevailing poverty. Simultaneously, poor adherence to unsupervised ambulatory treatment regimens fostered the development of increasing numbers of MDR-TB cases [12].

It was promptly noted however that there were no data on the magnitude of the problem. For this reason, WHO and the International Union Against Tuberculosis and Lung Disease (IUATLD) began the Global Project on Anti-tuberculosis Drug Resistance Surveillance in 1994. The study showed that the prevalence of multidrug resistance among new patients was generally low, the median value being $1 \%$. However, there were several "hot spots", i.e. countries or regions where the prevalence of multidrug resistance among new TB patients exceeded 3\%; a sobering finding was that drug resistance was found in all 72 countries included in the survey [14].

MDR-TB results from poor TB management; its prevalence is up to ten times higher in previously treated patients than in new patients. A poorly functioning TB program can create MDR-TB much faster than it can be treated, even if unlimited resources are available.

\section{The DOTS Strategy and the Standardized First Line Drugs Regimens}

The main selection factor for MDR-TB is inadequate drug treatment, which is prevented with the Directly
Observed Therapy-Short (DOTS) strategy in the treatment of new cases. Most countries of the world have now adopted the DOTS strategy, as recommended by the WHO. This strategy advocates standard initial 6-month regimens for new cases that include rifampin, isoniazid, ethambutol and pyrazinamide, and a reinforced regimen for previously treated cases [15]. This regimen was originally known as Category 1 treatment by the WHO (although the WHO does not use this term anymore, it is still known as Category 1 in many countries); for cases that are not cured with this regimen, the WHO recommends a reinforced first-line drugs regimen, also known as Category 2. This reinforced regimen includes streptomycin, isoniazid, rifampin, pyrazinamide, and ethambutol for 2 months, isoniazid, rifampin, pyrazinamide, and ethambutol for 1 month and isoniazid and rifampin plus ethambutol for 5 months. It is important to point out that this Category 2 regimen was never validated through a clinical trial and has been the motive of intense controversy and rejection by many countries and scientific societies because it favors many of the ideal conditions for drug resistance amplification in a drug resistant strain [1517]. In fact, the WHO does not recommend this regimen anymore for treatment failures, although it does recommend it for relapses and patients that have defaulted treatment. This regimen will certainly be phased out when every TB program around the world is capable of offering the appropriate strategies for diagnosis and treatment of retreatment cases presented in this review.

Overall, these standardized first line drugs regimens, especially the category 1 regimen, have proven to be effective in patients with drug-sensitive TB; in patients with drug-resistant strains, their efficacy is much lower, both in clinical trials and under program conditions [15].

In most low- and middle-income countries that have adopted the DOTS strategy, drug sensitivity testing is not performed for new cases or for most patients requiring retreatment [18]. Therefore, patients with underlying (and undetected) drug resistance will receive the same standardized treatments, and can be predicted to have higher rates of failure and relapse.

This leads to a major area of controversy in the treatment of MDR-TB: the use of standardized or individualized regimens to treat MDR-TB in countries with limited resources. In high-income countries with a low incidence of TB and sufficient financial, technical and human resources, MDR-TB treatment with individualized regimens is based on drug susceptibility tests.

Advocates of individualized treatment for the control of MDR-TB argue that empirical short-course chemotherapy regimens can amplify the problem of MDR-TB in patients already infected with strains resistant to one or more drugs [19]. In a recent ecological study [14] that included data from 103 countries, results show that, among the 20 countries where prevalence of initial multidrug resistance exceeded $3 \%$ and have used the standardized initial regimens previously mentioned, failure rates averaged $5.0 \%$, and relapse rates averaged $12.8 \%$, compared with an average of $1.6 \%(\mathrm{p}<0.0001)$ and $8.1 \%(\mathrm{p}<0.0002)$, respectively for the 83 countries where initial multidrug resistance prevalence was less than 3\%. In 92 countries using the standardized first line drugs retreatment regimen, failure rates were $2.7 \%$, 
$3.8 \%, 6.2 \%$, and $8.1 \%$ in quartiles of increasing prevalence of acquired multidrug resistance $(\mathrm{p}<0.0001)$. Failure and relapse with initial therapy, and the proportion of all patients treated who required first line drugs retreatment, were all significantly higher in countries where the prevalence of initial multidrug resistance exceeds $3 \%$, the WHO criterion for designation as an MDR-TB "hot zone". In all countries, first line drugs retreatment outcomes were poor-with high rates of default, failure, and mortality - which are important given the large number of patients requiring retreatment in all countries. These results suggest that the currently recommended regimens for previously untreated or new cases are adequate in countries where the prevalence of initial multidrug resistance is less than $3 \%$. But the findings raise concerns about using the same regimens in countries where initial multidrug resistance prevalence exceeded $3 \%$ [14]. These findings suggest that the standardized initial treatment for new cases should also be critically reexamined for these settings. In the long term, these standardized regimens may be contributing to amplification of multidrug resistance. The high rates of retreatment failure in countries with a more than $3 \%$ rate of initial multidrug resistance, and extremely high rates among patients who failed initial therapy in countries with high rates of acquired multidrug resistance, presumably reflect underlying multidrug resistance [14]. The continued use of the same regimen will result in unacceptably high rates of failure $[20,21]$, with amplification of resistance $[16,17]$. The standardized retreatment regimen requires urgent reappraisal because of very poor treatment outcomes - in all countries, but particularly in countries with higher prevalence of multidrug resistance.

Successful programs in both industrialized and developing countries indicate that a DOTS strategy when treating new cases prevents the appearance of MDR-TB. However, a common erroneous assumption is that DOTS by itself can reduce MDRTB once it has occurred. Actually there is no evidence that has causally linked the use of DOTS alone to observed declines of MDRTB. In fact, the claim that DOTS can reduce rates of MDR-TB is not supported by the large body of evidence that patients with MDR-TB have poor outcomes with short-course chemotherapy [22-27]. Untreated and untreatable sputum smear positive TB has a very high case fatality. However, patients may survive and disseminate drug resistant $M$. tuberculosis for years; if drug susceptible disease is treated and cured, resistant organisms will ultimately predominate. New York City is cited as an example of successful control of MDR-TB, yet this success cannot be attributed only to the strongly enforced directly observed treatment strategy; as important were the implementation of comprehensive interventions that included massive investments in infrastructure, infection control, improved case detection, and effective treatment of active cases of MDR-TB [13].

\section{WORLD EFFORTS TO CONTROL MDR-TB GLOBALLY}

In 1999, WHO created a working group on "DOTS-Plus for multidrug-resistant tuberculosis" to address the management of MDR-TB under program conditions [28]. This initiative was designed to assess the feasibility and cost- effectiveness of treating MDR-TB in low-income and middle-income countries. The WHO Green Light Committee, implemented in 2000 , serves as a technical advisory body to the Stop TB Partnership and WHO. Its primary tasks are: reviewing applications from countries that wish to benefit from quality-assured, second-line anti-TB drugs at reduced prices, promoting technical assistance to countries throughout the application and implementation processes and monitoring and evaluating GLC-approved programs to assess their progress and continued adherence to WHO guidelines; it also assists WHO with developing policy to control MDR-TB. It is comprised of representatives from institutions with specific programmatic, clinical, advocacy, and scientific and managerial expertise.

Many experts emphasize that effective treatment of new cases will prevent the emergence of MDR-TB, and for this reason, countries with limited resources should invest those scarce resources in treatment of drug susceptible cases, especially given that, even in high burden countries, MDR cases constitute a small proportion of total TB cases. Quite the opposite, other experts argue that it is unethical to abandon patients with MDR-TB and maintain that, if untreated, MDR-TB strains will become dominant and undermine global TB control [29] and that, to deny treatment to patients with MDR-TB is to violate their human rights [11]. Empirical evidence of the feasibility of treating MDRTB with a high rate of success in resource limited regions already exists. A pilot project involving community-based treatment of MDR-TB in northern Lima, Peru [30] proved that it was possible to cure MDR-TB on an outpatient basis in a country where $\mathrm{TB}$ was endemic, an experience that has now been replicated elsewhere [31]. However, it has been possible, in these isolated instances, to secure the resources needed to ensure compliance over the long period of treatment and manage the frequent side-effects of second line drugs. Unfortunately, a common mistake is to extrapolate results from a model, atypical region that has been successful in treating MDRTB to a whole country. Differences between these usually heavily funded and highly successful regions and the rest of the country are frequently greater than differences between countries [32].

Current strategies for MDR-TB control consider essentially the prevalence of primary multidrug resistance (MDR in new cases), but not the total burden in a country. In some high-prevalence countries, the proportion of retreatment cases is considerable and related to a high burden of multidrug resistance, despite primary drug resistance rates that are still low. For this reason, any control framework should take into account the prevalence of all drug resistant cases in order to make a realistic estimate of the overall problem in the community [33].

Taking into account all these factors, the essential components for the implementation of a DOTS-Plus strategy can then be summarized as follow:

1. An efficient, effective and integrated TB control program

2. First-line and second-line anti-TB drugs provided free of charge to each patient with MDR-TB

3. Early diagnosis and free treatment of antituberculosis drugs side effects 
4. Drug susceptibility tests for first line and second-line anti-TB drugs, not charged to the patients

5. Appropriately designed regimens for MDR-TB

6. A reporting system for data management, monitoring and evaluation of individual and aggregated data on MDR-TB cases

7. Community-based strategies, with the participation of local governments in order to enhance adherence to the regimens

8. The adequate training and organization of health professionals responsible for the care of MDR-TB patients

\section{TUBERCULOSIS AND MDR-TB: AN EXAMPLE OF SOCIAL INEQUITY}

TB is considered a sobering example of inequity. It is a disease predominantly of the socially and economically disadvantaged, since its incidence is exacerbated by socioeconomic factors, inconsistent and/or inadequate treatment practices and immigration from countries in which the disease is endemic $[34,35]$. Reducing inequalities in TB incidence in the world is, therefore, a goal that needs to be addressed, especially since the recent global financial crisis is expected to exacerbate these health inequalities [36, 37].

There is a strong negative association between Gross Domestic Product (GDP) per capita and TB outcomes, a finding in accordance with previous research on TB [38-41] and other health outcomes [34] (Fig. 1).
Despite the overall wealth and level of economic development within a nation, income inequality increases the numbers of individuals within a country that have limited access to healthcare. Another potential explanation of the effect of income inequality on TB is that income inequality results in increased urban poverty, which is associated with well-known risk factors for TB, such as overcrowding and polluted environments [34, 42]. The Gini coefficient is a measure of statistical dispersion that can be used to measure the inequality in the levels of income. A coefficient of zero would represent perfect equality where everyone would have exactly equal income while a coefficient of 100 expresses maximal inequality where only one person would have all the income. In general, countries with lower Gini coefficients have lower TB rates. As expected, countries with greater income inequality have higher TB rates (Fig. 2).

Political will must emerge to challenge the systematic problem of neglected diseases research, as the lack of a profitable market for diseases primarily affecting people in developing countries meant that research and development had come to a standstill for decades. Only $1 \%$ of the new medications developed between 1975 and 1999 were treatments for tuberculosis and tropical diseases, despite these diseases causing $11 \%$ of the global disease burden [43-45]. Developing countries are struggling with the growing and costly challenge of multidrug-resistant (MDR) and extensively drug-resistant TB [46]. Eliminating these disparities in the quality of tuberculosis care is the best way towards global TB control, and as a consequence of MDR-TB. Treatment of pansensitive TB costs less than \$10 US, while diagnosis testing and treatment of MDR costs run in the thousands of dollars.

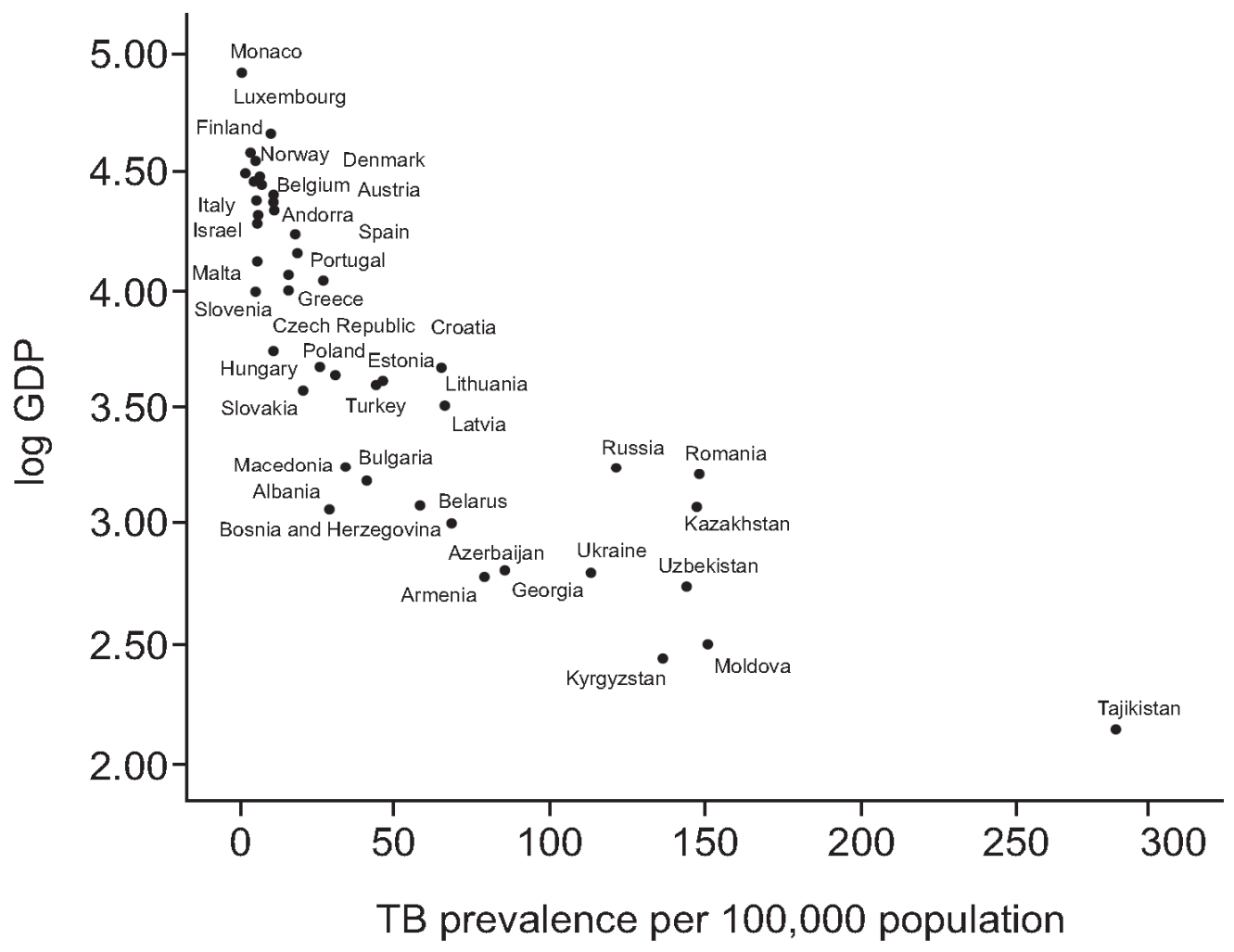

Fig. (1). Scatter plot of the association between the baseline (2000) logged gross domestic product (GDP) and tuberculosis (TB) prevalence for WHO Euro region in 2005 (reproduced with permission from the Eur Respir J 2012; 40: 925-930). 


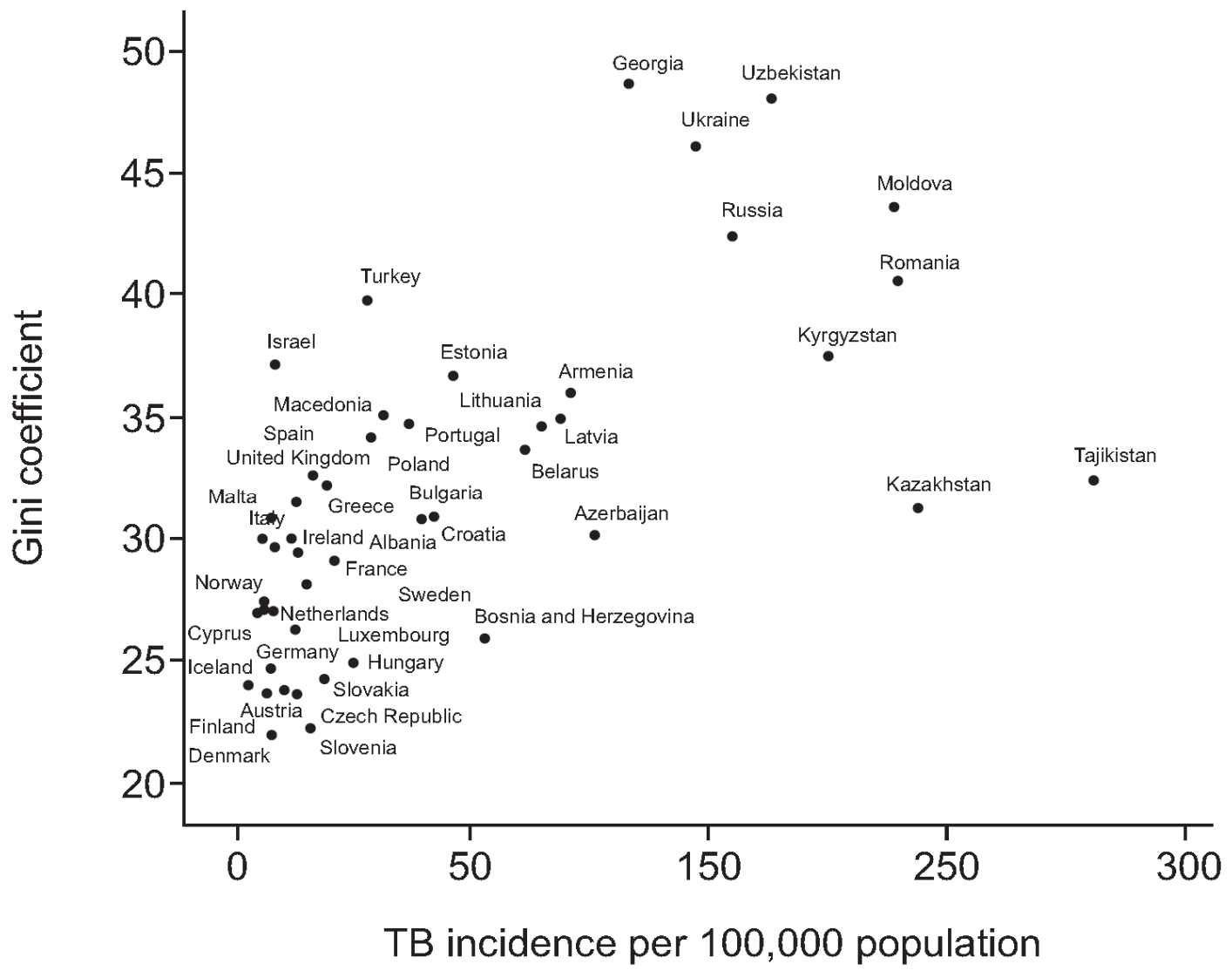

Fig. (2). Scatter plot of the association between baseline Gini coefficient2000 and tuberculosis (TB) incidence for WHO Euro region in 2004. (reproduced with permission from the Eur Respir J 2012; 40: 925-930).

Considerable efforts by civil society organizations in recent years have helped make MDR-TB a public health priority. As with HIV, it required challenging notions that MDR-TB was untreatable in resource-limited settings and that it would divert attention and resources from treating drug-susceptible TB. Although MDR-TB is now an international priority with an agreed-upon global plan to universal access to treatment [47], too little practical progress has been made over the last decade. It is ethically unacceptable that only $10 \%$ of the patients with MDR-TB are diagnosed and receive appropriate treatment under programmatic conditions [46]. There is also an unknown number of MDR-TB patients treated outside the scope of national TB programs, with unorthodox regimes and drugs of indefinite quality that increase the risk of developing more extensive drug resistance.

Most of the laboratory tests needed to diagnose MDR-TB requires specialized and very costly equipment and reagents; this constitutes an enormous obstacle for their implementation in developing countries, which as mentioned, usually have the highest burden of drug resistant TB cases. Also, the cost of second-line drugs remains high at around $\$ 4,500$ on average per patient. Currently, the MDRTB drug market is too small and fragmented and barely attracts manufacturers. Price reductions from economies of scale will be realized only when more patients are put on treatment, but the vicious circle of high costs dissuading countries from addressing MDRTB - meaning limited patient numbers keep prices high — needs to be overcome $[48,49]$.

In conclusion, poverty and socioeconomic inequalities are barriers that deny access to state of the art diagnostic methods and treatment for the majority of MDR-TB patients in an even greater degree than that for drug susceptible TB. As long as these inequities persist or at least are markedly reduced it will be impossible to achieve real global control of drug resistant tuberculosis.

\section{NEW CHALLENGES IN THE DIAGNOSIS AND TREATMENT OF MDR-TB}

\section{Drug Susceptibility Testing to Isoniazid and Rifampin for All TB Patients}

Definitive diagnosis of drug-resistant TB requires $M$. tuberculosis isolation and drug susceptibility testing (DST). The possibility of drug-resistant TB should be considered at the time of specimen collection; sputum or other specimens should be then processed for culture and DST. Ideally DST to isoniazid $(\mathrm{H})$ and rifampin $(\mathrm{R})$ should be carried out in every case diagnosed with TB including new cases; previously treated patients obviously constitute an urgent priority. Once resistance to $\mathrm{R}$ is proven, DST to antituberculosis fluorquinolones (FQ) and second line injectable agents should be the next diagnostic step. This would allow a more opportune diagnosis of XDR-TB and 
avoid the use of inefficient drug regimens that would only extend the degree of resistance.

\section{Decreasing Turnaround Times for Culture and DST Results}

Isolation in culture provides for all subsequent testing, including identification and antibiotic susceptibility assays. The time-honoured proportions method (Canetti, Rist, Grosset, 1963 [50] is still considered the gold standard for detection of drug resistance to first and second line drugs (FLD, SLD), with the exception of pirazinamide. Despite its high accuracy and reproducibility for $\mathrm{H}$ and $\mathrm{R}$, (and to a lesser scale for FQ and SLD, its prolonged turnaround time (60 to 120 days) constitutes a major disadvantage of this method. Even with direct inoculation from smear positive specimens $(2+$ or $3+)$, minimal turnaround time for detection of resistance to isoniazid and rifampin is about 30 days.

Clearly there was an urgent need to shorten the turnaround time for diagnosis of drug resistance in order to avoid the further clinical deterioration of MDR-TB cases being treated with inadequate FLD based regimens (generating even more extensive drug resistance) and what is more important from epidemiological point of view, to interrupt the chain of transmission of drug resistant strains in the community by reducing the time during which the patient is infectious; there is ample evidence that a patient with MDR-TB will remain infectious in spite of being treated with FLD regimens [51].

\section{Rapid Phenotypic DST}

The commercial system BACTED $460-\mathrm{TB}^{\circledR}$ was the first automated system available for mycobacterial testing [52]; it is a radiometric method that allows much shorter turnaround times in comparison with the proportions methods; it became the state of the art for DST during the 1990's, but its use has been now virtually abandoned due to the inconvenience of disposing of the radioactive $\mathrm{C}^{14}$ used in the assay. It was substituted by the BACTEC MGIT- $960^{\circledR}$ system, also an automated method with short turnaround times. The MGIT$960^{\circledR}$ has become a sort of standard method for detection of drug resistance to FLD, including pirazinamide. This method uses a liquid media (Middlebrook 7 H9) with a fluorescent sensor to detect bacterial growing. Depending of the concentration of mycobacteria in the sample cultivated, it takes an average of 3 weeks from time of inoculation to reporting of drug susceptibility $[52,53]$. Most often, the "indirect susceptibility method" is used, in which growth is first isolated in pure culture from clinical specimens, with subsequent inoculation of drug-containing broth. The direct inoculation of processed samples of AFB positive sputa allows the obtention of DST in an average of 11 days, saving at least a week of waiting time in relation to the conventional indirect inoculation [54-56]. However, direct testing of clinical specimens is problematic due to the potential for bacterial contaminants and other nontuberculous species, resulting in assay failure rates of up to $15 \%$. For this reason, most laboratories rely on the indirect method for susceptibility testing. One important limitation of these automated diagnostic systems is the high cost of the reagents.
There are more affordable phenotypic methods used mainly in developing countries, that WHO recently validated under stringent laboratory protocols in reference or national laboratories in selected settings [57]. These methods for rapid detection of resistance to isoniazid and rifampin include the MODS assay, the CRI (Colorimetric Redox Indicator) and nitrate reductase assay (Griess method). In spite of their economic advantage and in some cases technical simplicity, the time for diagnosis of DR-TB was not shortened substantially compared with other phenotypic methods but they still might be valuable tools in countries with limited resources for laboratory diagnosis of DR-TB.

It is important to point out that the reliability and reproducibility of phenotypic drug testing for most SLD (including ethionamide, prothionamide, cycloserine, terizidone, p-aminosalicylic acid, clofazimine, amoxicillinclavulanate, clarithromycin, linezolid) and even for FLD like ethambutol and pyrazinamide, is poor and varies from one laboratory to another [58].

It is also important to emphasize that even the fastest phenotypic DST systems will have a 10-15 day turnaround time in ideal conditions, and though faster than the traditional proportions method, is still far from being optimal for clinical decision making.

\section{Rapid Genotypic DST}

The development of genotypic methods generated a genuine revolution in the diagnosis of DR-TB. The polymerase chain reaction (PCR) based molecular methods (originally developed in 1983) allow for the specific identification of $M$. tuberculosis and the detection of drug resistance in a matter of hours instead of weeks. Line probe assays (LPAs) are based on reverse hybridization of oligonucleotides with specifically designed probes. Two methods are currently available for clinical use and results can be obtained within 24 hours: Line Probe Assay (LiPA) ${ }^{\circledR}$ and the more recent GenoTypeMTBDRplus and GenoTypeMTBDRsl $^{\circledR}$ (FLD and SLD respectively). GenoType MTBDRplus has the capacity to detect resistance to isoniazid and rifampin in clinical isolates and sputum samples based on the detection of the most frequent mutations associated to isoniazid and rifampin resistance (kat $G$, inh $A$ genes for isoniazid and rроB gene for rifampin). Sensitivity and specificity for detection of rifampin resistance is over $98 \%$ but slightly lower for the detection of isoniazid resistance due to the existence of other mutations associated with isoniazid resistance not detected by the assay. Also, a small proportion of strains resistant to rifampin harbour mutations different to that of the rpoB gen; resistance associated to those mutations will be detected through phenotypic methods and not by molecular techniques designed specifically to detect the rpoB mutation [59].

The GenoType MTBDRs $l^{\circledR}$ can detect resistance to fluoroquinolones, amikacin/capreomycin and ethambutol through mutations in the genes gyrA, rrs and embB. The overall sensitivity for fluoroquinolones, amikacin, capreomycin and ethambutol was, respectively $90.2 \%$, $83.3 \%, 86.8 \%$ and $59.0 \%$ with specificity close to $100 \%$ [59]. WHO does not recommend its use due to its low 
sensitivity for SLD; limitations for its use in resource constrained settings include the need of especially trained personnel, adequate installations and high cost of the assay. A comparative cost analysis was done in a developing country (Peru) among the MODS, Griess and Genotype MTBDRplus techniques; the estimated costs for each test were USD $\$ 5.33, \$ 5.58$ and $\$ 63.50$, respectively. In a programmatic level, the use of the Griess and MODS is overwhelmingly more economical than the Genotype, so the possibility of using this later assay would be very limited in high-burden regions and limited resources [60].

Recently a new technique, the $\mathrm{Xpert}^{\mathbb{B}}$ MTB/RIF has become clinically available; it was designed for point oftreatment use in low-income countries [61]. The XpertMTB/RIF (GeneXpert ${ }^{\circledR}$ system, Cepheid, Sunnyvale, California) is an automated molecular test for TB case detection and rifampin resistance. In a recent study the XpertMTB/RIF demonstrated sensitivities of $98.2 \%$ and $72.5 \%$ for detecting TB in smear-positive and smearnegative tuberculosis, respectively. The test's specificity was $99.2 \%$ in patients without TB. This new technology is very simple to use requiring only electricity and personnel with minimal training. Is based on a single-use sample-processing cartridge that integrates real-time PCR technology; the assay requires a single manual step with minimal sample manipulation (sputum liquefaction/inactivation with a reagent and its transfer into the test cartridge). The analysis is completed automatically by the instrument in about 2 hours [61]. The method was endorsed by WHO in December 2010 and a policy statement was released in 2011 [62]. The negotiated prices between international donors, a non profit organization and the producer, has reduced the cost per test to USD \$9.98 for public institutions from a list of 145 countries with high burden of MDR-TB and low income economies. A four cartridge machine has a cost of $\sim \mathrm{USD}$ $\$ 17,000$ plus the costs of yearly manteinance $[63,64]$.

The XpertMTB/Ri ${ }^{\oplus}$ opens the possibility for costeffective point of-treatment application in developing countries where MDR-TB prevalence is high and resistance to rifampin a strong predictor of MDR-TB. For example, in a patient with a history of FLD treatment for TB, and symptoms suggestive of relapse, the XpertMTB/Rif ${ }^{\oplus}$ in just two hours after the sputum sample is delivered to the laboratory, could provide the physician with information regarding the presence of $M$. tuberculosis and its sensitivity or resistance to rifampin. If the strain was resistant the patient would not be treated again with the same FLD regimen received in the past, due to the high probability of harbouring an MDR strain; depending on the patient clinical status, he or she could either start immediately with an empirical SLD regimen or wait for the definitive DST to be completed (by phenotypic or genotypic methods) to design and individualized regimen.

The main limitations of the $\mathrm{Xpert}^{\circledR}$ are: a) it only detects resistance to rifampin (will also not detect those mutations that occur outside of the rpoB core region, mutations that amount to $\sim 5 \%$ of total mutations that confer resistance to rifampin), b) its lower capacity of detection when the prevalence of rifampin resistance is low, although sensitivity has improving in the new version of the Xpert ${ }^{\Theta}$; and c) the cost and some practical issues to be considered in developing countries such as electricity supply and air conditioning (required for proper operation of the device).

In conclusion, there are now a variety of methods available for rapid diagnosis of DR-TB with an ample range of costs and detection times. Molecular methods are the fastest but also the most expensive. It may be necessary for each high burden country to evaluate the cost-benefit of implementing molecular methods that though costly, can significantly reduce the time to diagnosis compared with conventional methods; as a consequence this will curtail the transmission of drug resistant strains in the community, a fundamental element towards the control of drug resistant tuberculosis.

\section{CHALLENGES IN THE TREATMENT OF MDR-TB}

As is well known, tuberculosis treatment must be based on two significant bacteriological considerations: regimens that include multiple drugs to avoid the selection of resistant mycobacteria and the need for prolonged duration of treatment to ensure the elimination of all bacteria, regardless of their metabolic rate (active or latent). Treatment of MDRTB then must include at least 4 and up to 5 drugs that has never been used by the patient and/or have proven to be effective by reliable DST's. The regimen should include drugs in an order based on their bactericidal and sterilizing profile as well as their potential toxicity. Antituberculosis drugs are traditionally classified in 5 groups (Table 1) [65].

Table 1. Antituberculosis Drugs with Potential Activity in MDR-TB

\begin{tabular}{|l|l|l|}
\hline Group 1 & First line drugs & ethambutol, pirazinamide \\
\hline Group 2 & Fluoroquinolones & levofloxacin, moxifloxacin \\
\hline Group 3 & Injectable agents & kamamycin, amikacin, capreomycin \\
\hline Group 4 & $\begin{array}{l}\text { Bacteriostatic oral } \\
\text { agents }\end{array}$ & $\begin{array}{l}\text { cycloserine/terizidon, } \\
\text { ethionamide/protionamide, p- } \\
\text { aminosalicylic acid (PAS) }\end{array}$ \\
\hline Group 5 & $\begin{array}{l}\text { Miscellaneous } \\
\text { agents (debated } \\
\text { efficacy) }\end{array}$ & $\begin{array}{l}\text { clofazimine, linezolid, amoxicillin- } \\
\text { clavulanate, imipenem/cilastatin, } \\
\text { meropenem/clavulanate, high dose } \\
\text { isoniazid }\end{array}$ \\
\hline
\end{tabular}

First line drugs (Group 1) that are still effective according to DST's will be included (basically pyrazinamide and ethambutol), then only one drug from both group 2 (fluorquinolones) and group 3 (SLD injectables) will be added since drugs in those groups share bacterial target sites of action and frequently have cross resistance with drugs within the group. Every MDR-TB treatment regimen should include a new generation fluorquinolone (high doses of either levofloxacin or moxifloxacin) unless there is proof of resistance; is important to emphasize however, that although class cross resistance is common for fluoroquinolones and injectables, it not infrequent that a MTB strain will be resistant to one fluorquinolone or injectable but susceptible to another. If we need more drugs to create the regimen we then choose a drug from group 4 (oral second line agents); finally, if it is absolutely necessary we can include drugs from group 5, for which (except for thiacetazone) there are no clinical trials data to document their efficacy; this has been reported only from in vitro studies or animal models. 
For this reason, drugs from group 5 have been designated as reserve drugs due to their low antituberculosis activity and frequent adverse reactions and toxicity.

An important issue regarding treatment for MDR-TB is that a drug that is been employed in a regimen that is failing should not be included in the new regimen even if in the DST's the strain is reported as susceptible. In that case the drug could be used as an additional drug, but not count as one of the four effective drugs that the regimen must include. There is a current trend that favours the inclusion of pyrazinamide in MDR-TB regimens. The theoretical basis for its use is that pyrazinamide is always used under the "protection" of $\mathrm{H}$ and $\mathrm{R}$ in FLD regimens and then only for two months, usually before the emergence of resistance. Although this might sound as a solid argument, confirmation of drug susceptibility is complex or unreliable, even with the automated methods. Therefore if pyrazinamide is added to the regimen it should not be counted as part of the minimal number of effective drugs.

Currently, treatment strategy for MDR-TB offers two general approaches to treatment on the basis of existing program capabilities:

- Standardized treatment in which all patients with MDR-TB receive the same treatment regimen derived from predominant patterns of resistance in the community in the absence of a complete panel of drug susceptibility tests for given case. An alternative to this approach is an individually designed empirical treatment based on the patient's previous history of anti-TB treatment with consideration of DST data from a representative patient population

- Individualized treatment in which patients with MDR-TB receive culture-based DST, and treatment regimens are then tailored accordingly

Regardless of which strategy is used, the therapeutic cornerstone in MDR-TB is the combination of an injectable agent and a fluoroquinolone plus at least 2 or 3 drugs with potential activity according to treatment drug history and DST results. As mentioned, pyrazinamide could be considered as an add on, but not count as one of the minimal number of effective drugs.

A typical standardized regimen for MDR-TB comprises an initial phase of at least 6 months, or until 4 months after culture conversion, comprising an injectable (kanamycin/amikacin or capreomycin) plus a fluoroquinolone (levo or moxifloxacin) associated to ethionamide, pyrazinamide and cycloserine or PAS, followed by a continuation phase with the oral agents for about 12 to 18 months [65].

\section{NEW ANTITUBERCULOSIS DRUGS}

Despite the enormous number cases of TB worldwide, the therapeutic arsenal to treat this disease continues to be very limited, especially for cases with extensive drug resistance. One of the reasons for this situation was that in the 1970's unfortunately it was though without good data that $\mathrm{TB}$, as with other infectious diseases, was on the brink of being eliminated and drug development research was not a priority anymore.
For the first time in decades, the pipeline of new anti-TB agents is now growing again. New drugs and combination of drugs with interesting potential efficacy to treat TB, MDRTB and XDR-TB have appeared during the last few years.

Tsukamura's published in 1985 [66] the antituberculosis activity of the fluorated quinolone ofloxacin in patients with intractable TB: it was the first on a class of potent anti-TB drugs that inhibit the bacterial DNA gyrase, thereby inhibiting DNA transcription and replication. Twenty years later, Andries reported a new drug, derived from antimalarial quinolines that targeted the proton pump related to ATP synthase of M. tuberculosis [67]. The compound originally denominated R207910, then TMC207 and finally bedaquiline, showed early on a promissory activity against $\mathrm{TB}$ and $\mathrm{DR}-\mathrm{TB}$, although its interaction with rifampin precludes its use in combination with the most important FLD drug for pan-susceptible TB [68].

In-between these two important developments, an oxazolidinone called linezolid (the first drug in this category was cycloserine, discovered in 1955) was released in 2000 for the treatment of Gram positive infections (mainly Staphylococcus aureus) and was utilized off-label for MDRTB. Linezolid inhibits protein synthesis, and has good activity against multidrug-resistant $M$. tuberculosis, but its potential toxicity and elevated cost makes it almost unaffordable in developing countries, where is mainly used in XDR-TB cases [69] Reduced dosage of linezolid (600 $\mathrm{mg}$ /day and even a quarter of the regular dose, $300 \mathrm{mg}$ a day) has still shown efficacy in treatment regimen for MDR and XDR-TB; this obviously would reduce the overall cost of treatment and make linezolid more affordable [70, 71]. Recently in India, patients with MDR and XDR-TB where successfully treated $(89.7 \%$ culture conversion rate) with a generic, locally produced linezolid (cost per tablet less than $\$ 1$ USD [72]. New oxazolidinones (PNU-100480 [sutezolid] and AZD-5847 [posizolid]) with an expected lower toxicity and even greater efficacy are under initial human studies [73, 74].

This decade has witnessed the rediscovery of old drugs with potential activity against DR-TB. Clofazimine, a very useful drug in the treatment of leprosy was rediscovered as a potential effective anti-TB drug. A systematic review recently published including 3,489 MDR-TB patients from 10 different countries, showed that treatment regimens including clofazimine have had a pooled treatment success of $62 \%$ [75]. However, it is almost impossible to establish the individual efficacy of any drug from retrospective studies that analyze treatment regimens that include multiple agents. The so called "Bangladesh study" showed the effectiveness ( $87.9 \%$ relapse free after 2 years of follow-up) of a shortened regimen for MDR-TB including clofazimine plus gatifloxacin, ethionamide, pirazinamide, high dose isoniazid, ethambutol and kanamycin [76]. Obviously, before this shortened regimen is considered for widespread use, more evidence is needed.

The WHO's group $\mathrm{V}$ of drugs include among others drugs like carbapenems (imipenem, meropenem) and clavulanate that have moderate activity against $M$. tuberculosis in the murine model [77]. A small clinical study that included XDR-TB patients with a history of multiple treatment regimens, showed some efficacy of salvage 
regimens that included meropenem and clavulanate [78]. There is a report of a paediatric XDR-TB case successfully treated with the association of meropenem-clavulanate and linezolid [79].

Other compounds with clinical potential of efficacy in TB treatment in the near future are the nitroimidazopyrans, compounds derived from metronidazole. Particularly two molecules, PA-824 [80] and OPC-6783 (delamanid) [81] are currently evaluated in Phase III clinical studies.

Another promising compound is SQ109, a drug derived from ethambutol; it has a similar mechanism of action (interferes with the synthesis of the bacterial cell wall) but without cross resistance with it. SQ109 has shown in vitro synergy with another compound, PNU-100480 [82]. Clinical trials are just getting underway for this drug.

An interesting, though controversial approach in tuberculosis treatment is the use in limited clinical trials, based on results in animal model, of combinations of new or new and older drugs, with the objective of increasing the bactericidal activity of the regimen. A murine model measuring sterilizing activity and relapse rate showed that the combination of bedaquiline, PA-824, PNU-100480, and/or clofazimine proved to be superior to the first-line regimen of rifampin, pyrazinamide, and isoniazid. The best combination in this study was that of bedaquiline-PNU100480. This type of combinations could eventually become an effective "universal" standardized regimen for DR-TB [83].

In another prospective, early bactericidal activity (EBA) study that included new compounds, 80 treatment-naive, drug-susceptible patients with uncomplicated pulmonary TB, were randomized into 6 groups. During 14 days subjects were to receive one of the following regimens: bedaquiline, bedaquiline-pyrazinamide, PA-824-pyrazinamide, bedaquilinePA-824, PA-824-moxifloxacin-pyrazinamide, or unmasked standard antituberculosis as control. The maximum EBA was achieved by the combination of PA-824-moxifloxacinpyrazinamide. If further proven on larger scale, this kind of regimens may be suitable for treating both drug-sensitive and drug-resistant tuberculosis [84]. The goal of developing a regimen effective on drug-susceptible and drug-resistant $M$. tuberculosis would allow controlling both forms of the disease with a single treatment, a desirable goal in resource limited regions, where availability of DST are limited or non-existent. A foreseeable problem with this approach is the virtually unavoidable emergence of mycobacterial resistance after the introduction of any new antibacterial drug.

In conclusion, new and encouraging developments have appeared in recent years in the pharmacological horizon for the treatment of tuberculosis including DR-TB [85]. Strong international mobilization and political will drove a golden decade for global health; however, donor support has started to decline at a time when many patients still wait for treatment and the prices of needed newer medicines are on the increase due to patent protection [43]. The development of new anti-TB drugs has been slowed down by several obstacles. First of all, the TB drug market is associated with insufficient profit opportunity or investment return to motivate pharmaceutical companies to develop new drugs when the cost of developing a new drug is estimated at $\$ 115$ to $\$ 240$ million USD [86]. To be profitable, market prices of new drugs must be relatively high, whereas the cost of the standard regimen is only about $\$ 11$ USD per patient [87].

\section{CONFLICT OF INTEREST}

The authors confirm that this article content has no conflicts of interest.

\section{ACKNOWLEDGEMENTS}

Declared none.

\section{REFERENCES}

[1] Pablos-Méndez A, Gowda DK, Frieden TR. Controlling multidrugresistant tuberculosis and access to expensive drugs: a rational framework. Bull World Health Org 2002; 80: 489-500.

[2] Global tuberculosis control: WHO report 2011. WHO Library Cataloguing-in-Publication Data ISBN 9789241564380

[3] Zignol M, Hosseini MS, Wright A, et al. Global incidence of multidrug-resistant tuberculosis. J Infect Dis 2006; 194: 479-85.

[4] Anti-Tuberculosis Drug Resistance in the World: Fourth Global Report. Geneva: World Health Organization; 2008. WHO/IUATLD Global Project on Anti-Tuberculosis Drug Resistance Surveillance.

[5] Raviglione M, Marais B, Floyd K, et al. Scaling up interventions to achieve global tuberculosis control: progress and new developments. Lancet 2012; 379: 1902-13.

[6] Global tuberculosis control: WHO report 2012. WHO Library Cataloguing-in-Publication Data ISBN 9789241564502

[7] Migliori GB, Sotgiu G, Gandhi NR, et al. Drug resistance beyond XDR-TB: results from a large individual patient data metaanalysis. Eur Respir J 2012 Oct 11. [Epub ahead of print]

[8] Migliori GB, Centis R, D’Ambrosio L, et al. Totally drug-resistant and extremely drug-resistant tuberculosis: the same disease? Clin Infect Dis 2012; 54: 1379-80.

[9] Migliori GB, Sotgiu G. Treatment of tuberculosis: have we turned the corner? Lancet 2012; 380: 955-7.

[10] Fox W, Mitchison DA. Short-course chemotherapy for pulmonary tuberculosis. Am Rev Respir Dis 1975; 111: 325-353.

[11] Hong Kong Tuberculosis Treatment Services- British Medical Research. A study in Hong Kong to evaluate the role of pretreatment susceptibility tests in the selection of regimens of chemotherapy for pulmonary tuberculosis. Am Rev Respir Dis 1972; 106: 1-22.

[12] Frieden TR, Sterling T, Pablos-Méndez A, et al. The emergence of drug-resistant tuberculosis in New York City. New Eng J Med 1993; 328:521-526.

[13] Suárez PG. First requirement for control of multidrugresistant TB: realism. Bull World Health Org 2002; 80: 496.

[14] Zignol M, van Gemert W, Falzon D, et al. Surveillance of antituberculosis drug resistance in the world: an updated analysis, 2007-2010. Bull World Health Organ 2012; 90: 111-9.

[15] Mak A, Thomas A, del Granado M, et al. Influence of Multidrug Resistance on Tuberculosis Treatment Outcomes with Standardized Regimens Am J Respir Crit Care Med 2008; 178: 306-12.

[16] Espinal MA, Kim SJ, Suarez PG, et al. Standard short-course chemotherapy for drug-resistant tuberculosis: treatment outcomes in 6 countries. JAMA 2000; 283: 2537-45.

[17] Espinal MA. Time to abandon the standard retreatment regimen with first-line drugs for failures of standard treatment. Int J Tuberc Lung Dis 2003; 7: 607-08.

[18] Quy HT, Lan NT, Borgdorff MW, et al. Drug resistance among failure and relapse cases of tuberculosis: is the standard retreatment regimen adequate? Int J Tuberc Lung Dis 2003; 7: 631-36.

[19] World Health Organization. Treatment of tuberculosis: guidelines for national programmes. Geneva: World Health Organization; 2003. WHO Publication No. WHO/CDS/TB/2003.313.

[20] Laniado-Laborín R. Multidrug-resistant tuberculosis: standardized or individualized treatment? The question has already been answered. Expert Rev Resp Med 2010; 4: 143-6.

[21] Fox W, Ellard GA, Mitchison DA. Studies on the treatment of tuberculosis undertaken by the British Medical Research Council 
tuberculosis units, 1946-1986, with relevant subsequent publications. Int J Tuberc Lung Dis 1999; 3: S231-S279.

[22] Saravia JC, Appleton SC, Rich ML, et al. Retreatment management strategies when first-line tuberculosis therapy fails. Int $\mathrm{J}$ Tuberc Lung Dis 2005; 9: 421-9.

[23] Kim JY, Mitnick CD, Bayona J, et al. Examining assumptions about multidrugresistant TB control. World Health Org 2002, 80: 498-99.

[24] Mitchison DA, Nunn AJ. Influence of initial drug resistance on the response to short-course chemotherapy of pulmonary tuberculosis. American Review of Respiratory Disease 1986; 133: 423-30.

[25] Manalo F, Tan F, Sbarbaro JA, Iseman MD. Community-based short-course treatment of pulmonary tuberculosis in a developing nation. Initial report of an eight-month, largely intermittent regimen in a population with a high prevalence of drug resistance. American Review of Respiratory Disease 1990; 142: 1301-5.

[26] Kimerling ME, Kluge $\mathrm{H}$, Vezhnina $\mathrm{N}$, et al. Inadequacy of the current WHO re-treatment regimen in a central Siberian prison: treatment failure and MDR-TB. Int J Tuberc Lung Dis1999; 3: 451-3.

[27] Centers for Disease Control and Prevention. Primary multidrugresistant tuberculosis-Ivanovo Oblast, Russia, 1999. Morbidity and Mortality Weekly Report 1999; 48: 661-3.

[28] World Health Organization. Guidelines for the programmatic management of drug-resistant tuberculosis. Emergency update. WHO/HTM/TB/2008.402. WHO, Geneva, Switzerland (2008).

[29] Farmer P, Kim JY. Community based approaches to the control of multidrug resistant tuberculosis: introducing "DOTS-plus". BM J 1999; 317: 671-674.

[30] Farmer P, Becerra M, Kim J, editors. The global impact of drugresistant tuberculosis. Boston, (MA): Harvard Medical School and Open Society Institute; 1999.

[31] Laniado-Laborín R, Estrada-Guzman J, Perez H, et al. Treatment of multidrug-resistant tuberculosis in a high-prevalence region through a binational consortium. Int J Tuberc Lung Dis 2012; 16: 610-1.

[32] Farmer P, Bayona J, Becerra M, et al. The dilemma of multidrugresistant tuberculosis in the global era. International Journal of Tuberculosis and Lung Disease 1998; 2: 869-76.

[33] Cullinan T. Multidrug-resistant TB-unexamined costs and complexities. Bull World Health Org 2002, 80: 496-7.

[34] Rieder HL, Lambregts-van Weezenbeek CSB. Sustainable TB control: the questions that have to be answered. Bull World Health Org 2002; 80: 499-500.

[35] Ploubidis GB, Palmer MJ, Blackmore C, et al. Social determinants of tuberculosis in Europe: a prospective ecological study. Eur Respir J 2012; 40: 925-30.

[36] Semenza JC, Giesecke J. Intervening to reduce inequalities in infections in Europe. Am J Public Health 2008; 98: 787-92.

[37] Rechel B, Suhrcke M, Tsolova S, et al. Economic crisis and communicable disease control in Europe: a scoping study among national experts. Health Policy 2011; 103: 168-75.

[38] Suhrcke M, Stuckler D, Suk JE, et al. The impact of economic crises on communicable disease transmission and control: a systematic review of the evidence. PLoS One 2011; 6: e20724.

[39] Beckfield J. Does income inequality harm health? New crossnational evidence. J Health Socl Behav 2004; 45: 231-48.

[40] Suk JE, Manissero D, Buscher G, et al. Wealth inequality and tuberculosis elimination in Europe. Emerg Infect Dis 2009; 15: 1812-14.

[41] Biggs B, King L, Basu S, et al. Is wealthier always healthier? The impact of national income level, inequality, and poverty on public health in Latin America. Soc Sci Med 2010; 71: 266-73.

[42] Morris JN, Wilkinson P, Dangour AD, et al. Defining a minimum income for healthy living (MIHL): older age, England. Int $\mathbf{J}$ Epidemiol 2007; 36: 1300-07.

[43] Godfrey R, Julien M. Urbanisation and health. Clin Med 2005; 5: $137-41$.

[44] von Schoen-Angerer T, Ford N, Arkinstall J, MA. Access to Medicines in Resource-limited Settings: The End of a Golden Decade? Global Adv Health Med. 2012; 1: 52-59.

[45] Trouiller P, Olliaro P, Torreele E, et al. Drug development for neglected diseases: a deficient market and a public-health policy failure. Lancet 2002 Jun 22; 359: 2188-94.
[46] Ma Z, Lienhardt C, McIlleron H, et al. Global tuberculosis drug development pipeline: the need and the reality. Lancet 2010; 375(9731): 2100-9.

[47] World Health Organization. Global tuberculosis control 2011. Geneva: World Health Organization; 2011.

[48] Nathanson E, Nunn P, Uplekar M, et al. MDR tuberculosiscritical steps for prevention and control. N Engl J Med 2010; 363: 1050-8.

[49] Médecins Sans Frontières. DR-TB drugs under the microscope: the sources and prices for drug resistant tuberculosis medicines. Geneva: Médecins Sans Frontières, International Union Against TB and Lung Disease; 2011.

[50] Canetti G, Rist N, Grosset J. Measurement of sensitivity of the tuberculous bacillus to antibacillary drugs by the method of proportions. Methodology, resistance criteria, results and interpretation. Rev Tuberc Pneumol (Paris) 1963; 27: 217-72.

[51] Escombe AR, Moore DA, Gilman RH, et al. The infectiousness of tuberculosis patients coinfected with HIV. PloS Med 2008; 5 (9): e188.

[52] Siddiqi SH, Libonati JP, Middlebrook G. Evaluation of a rapid radiometric method for drug susceptibility testing of Mycobacterium tuberculosis. J Clin Microbiol 1981; 13: 908-912.

[53] Van Deun A, Martin A, Palomino JC. Diagnosis of drug-resistant tuberculosis: reliability and rapidity of detection. Int J Tuberc Lung Dis 2012; 14: 131-40.

[54] Siddiqi S, Ahmed A, Asif S, et al. Direct drug susceptibility testing of Mycobacterium tuberculosis for rapid detection of multidrug resistance using the Bactec MGIT 960 system: a multicenter study. J Clin Microbiol 2012; 50: 435-40.

[55] Parrish NM, Carroll KC. Role of the Clinical Mycobacteriology Laboratory in Diagnosis and Management of Tuberculosis in LowPrevalence Settings. J Clin Microbiol 2011; 49: 772-6.

[56] Curry International Tuberculosis Center and California Department of Public Health, 2011: Drug-Resistant Tuberculosis: A Survival Guide for Clinicians, Second Edition.

[57] WHO. Policy statement on non-commercial culture and drug susceptibility testing methods for rapid screening of patients at risk for multidrug-resistant tuberculosis. Geneva, 2010. Available at: http://www.who.int/tb/laboratory/policy statements/en/index.html.

[58] World Health Organization (WHO). Policy Framework for Implementing New Tuberculosis Diagnostics, 2010. Available at: http://www.who.int/tb/laboratory/whopolicyframework_rev_june20 11.pdf.

[59] Palomino JC. Current developments and future perspectives for TB diagnostics. Future Microbiol 2012; 7: 59-71.

[60] Hillemann D, Rusch-Gerdes S, Richter E. Feasibility of the GenoTypeMTBDRsl assay for fluoroquinolone, amikacincapreomycin, and ethambutol resistance testing of Mycobacterium tuberculosis strains and clinical specimens. J Clin Microbiol 2009; 47: 1767-72.

[61] Solari L, Gutiérrez A, Suárez C, et al. Cost analysis of rapid methods for diagnosis of multidrug resistant tuberculosis in different epidemiologic groups in Peru. Rev Peru Med Exp Salud Publica 2011; 28: 426-31.

[62] Boehme CC, Nabeta P, Hillemann D, et al. Rapid molecular detection of tuberculosis and rifampin resistance. $\mathrm{N}$ Eng $\mathrm{J}$ Med 2010; 363:1005-15.

[63] WHO. Policy statement: automated real-time nucleic acid amplification technology for rapid and simultaneous detection of tuberculosis and rifampin resistance: Xpert MTB/RIF system. $\mathrm{WHO} / \mathrm{HTM} / \mathrm{TB} / 2011.4$

[64] Foundation for innovative new diagnostics (FIND). Negotiated prices for Xpert ${ }^{\circledR}$ MTB/RIF and FIND country list. August 2012 Available

http://www.finddiagnostics.org/about/what_we_do/successes/findnegotiated-prices/xpert_mtb_rif.html.

[65] WHO. Guidelines for the programmatic management of drugresistant tuberculosis. WHO/HTM/TB/2008.402

[66] Tsukamura M, Nakamura E, Yoshii S, et al. Therapeutic effect of a new antibacterial substance ofloxacin (DL8280) on pulmonary tuberculosis. Am Rev Respir Dis 1985; 131: 352-6.

[67] Andries K, Verhasselt P, Guillemont J, et al. A diarylquinoline drug active on the ATP synthase of Mycobacterium tuberculosis. Science 2005; 307: 223-7. 
[68] Mateello A, Carvalho ACC, Dooley KE, et al. TMC207: the first compound of a new class of potent antituberculosis drugs. Future Microbiol 2010; 5: 849-58.

[69] Grosset JH, Singer GT, Bishai WR. New drugs for the treatment of tuberculosis: hope and reality. Int J Tub Lung Dis 2012; 16: 100514.

[70] Sotgiu G, Centis R, D'Ambrosio L, et al. Efficacy, safety and tolerability of linezolid containing regimens in treating MDR-TB and XDR-TB: systematic review and meta-analysis. Eur Respir J 2012 Apr 10. [Epub ahead of print]

[71] Koh WJ, Kang YR, Jeon K, et al. Daily $300 \mathrm{mg}$ dose of linezolid for multidrug-resistant and extensively drug-resistant tuberculosis: updated analysis of 51 patients. J Antimicrob Chemother 2012; 67: 1503-7.

[72] Singla R, Caminero JA, Jaiswal A, et al. Linezolid: an effective, safe and cheap drug for patients failing multidrug-resistant tuberculosis treatment in India. Eur Respir J 2012; 39: 956-62.

[73] Wallis RS, Jakubiec WM, Kumar V, et al. Biomarker-assisted dose selection for safety and efficacy in early development of PNU100480 for tuberculosis. Antimicrob Agents Chemother 2011; 55: $567-74$.

[74] Shaw KJ, Barbachyn MR. The oxazolidinones: past, present, and future. Ann N Y Acad Sci 2011; 1241: 48-70.

[75] Dey T, Brigden G, Cox H, et al. Outcomes of clofazimine for the treatment of drug-resistant tuberculosis: a systematic review and meta-analysis. J Antimicrob Chemother 2012 Oct 10. [Epub ahead of print]

[76] Van Deun A, Maug AK, Salim MA, et al. Short, highly effective, and inexpensive standardized treatment of multidrug-resistant tuberculosis. Am J Respir Crit Care Med 2010; 182: 684-92.

[77] England K, Boshoff HI, Arora K, et al. Meropenem-clavulanic acid shows activity against Mycobacterium tuberculosis in vivo. Antimicrob Agents Chemother 2012; 56: 3384-7.
[78] Payen MC, De Wit S, Martin C, et al. Clinical use of the meropenem-clavulanate combination for extensively drug-resistant tuberculosis. Int J Tuberc Lung Dis 2012; 16: 558-60.

[79] Dauby N, Muylle I, Mouchet F, et al. Meropenem/clavulanate and linezolid treatment for extensively drug-resistant tuberculosis. Pediatr Infect Dis J 2011; 30: 812-3.

[80] Diacon AH, Dawson R, du Bois J, et al. Phase II dose-ranging trial of the early bactericidal activity of PA-824. Antimicrob Agents Chemother 2012; 56: 3027-31.

[81] Gler MT, Skripconoka V, Sanchez-Garavito E, et al. Delamanid for multidrug-resistant pulmonary tuberculosis. N Engl J Med 2012; 366: 2151-60.

[82] Reddy VM, Dubuisson T, Einck L, et al. SQ109 and PNU-100480 interact to kill Mycobacterium tuberculosis in vitro. J Antimicrob Chemother 2012; 67: 1163-6.

[83] Williams K, Minkowski A, Amoabeng O, et al. Sterilizng avtivities of novel combinations lacking first- and second-line drugs in a murine model of tuberculosis. Antimicrob Agents Chemother 2012; 56: $3114-20$

[84] Diacon AH, Dawson R, von Groote-Bidlingmaier F, et al. 14-day bactericidal activity of PA-824, bedaquiline, pyrazinamide, and moxifloxacin combinations: a randomised trial. Lancet 2012; 380: 986-93.

[85] Dooley KE, Obuku EA, Durakovic N, et al. World Health Organization Group 5 Drugs for the Treatment of Drug-Resistant Tuberculosis: Unclear Efficacy or Untapped Potential? J Infect Dis. 2012 Aug 30. [Epub ahead of print].

[86] Gardner CA, Acharya T, Pablos-Mendez A. The global alliance for tuberculosis drug development-accomplishments and future directions. Clin Chest Med 2005; 26: 341-7.

[87] van den Boogaard J, Kibiki GS, Kisanga ER, et al. New Drugs against Tuberculosis: Problems, Progress, and Evaluation of Agents in Clinical Development. Antimicrob Agents Chemotherapy 2009; 53: $849-62$. 